\title{
Diagnostic performance of treadmill exercise cardiac magnetic resonance: the prospective, multicenter EXACT trial
}

\author{
Subha V Raman ${ }^{*}$, Jennifer A Dickerson ${ }^{1}$, Wojciech Mazur ${ }^{2}$, Timothy C Wong ${ }^{3}$, Erik B Schelbert ${ }^{3}$, Debbie Scandling ${ }^{1}$ \\ , Jason Craft ${ }^{1}$, James Min ${ }^{4}$, Cheryl Bartone ${ }^{2}$, Ernest L Mazzaferri ${ }^{1}$, Paaladinesh Thavendiranathan ${ }^{1}$, John Arnold ${ }^{1}$, \\ Robert Gilkeson ${ }^{5}$, Orlando P Simonetti ${ }^{1}$
}

From 19th Annual SCMR Scientific Sessions

Los Angeles, CA, USA. 27-30 January 2016

\section{Background}

Stress cardiac magnetic resonance (CMR) typically involves pharmacologic agents that do not replicate symptoms and signs during typical exertion. Recent studies suggest excellent diagnostic performance of pharmacologic stress perfusion CMR compared to nuclear scintigraphy (SPECT); however, comparison of treadmill exercise stress CMR to SPECT has not been done.

\section{Methods}

Patients clinically referred for treadmill stress SPECT for the evaluation of known or suspected CAD were prospectively enrolled across 4 centers. After rest Tc99m SPECT imaging, patients underwent resting cine CMR. In-room stress was then performed using an MR-compatible treadmill with continuous 12-lead ECG monitoring and the Bruce exercise protocol. At peak stress, Tc99m was injected and patients were rapidly returned to their prior position in the magnet for real-time, free-breathing post-exercise cine and perfusion CMR. Following recovery monitoring with the table brought outside of the magnet bore, recovery cine and rest perfusion followed by late gadolinium enhancement acquisitions concluded the CMR portion of the exam. Stress SPECT images were then acquired in the adjacent nuclear laboratory. Patients not referred for invasive coronary angiography (ICA) within 2 weeks of stress imaging underwent coronary angiography with computed tomography (CTA). Diagnostic accuracy and prognostic value of treadmill stress CMR vs. SPECT were evaluated.

\section{Results}

Of 210 patients (age $57 \pm 11$ years, $40 \%$ female) completing the study protocol, exercise time averaged $9.0 \pm$ 2.6 min of the Bruce treadmill protocol achieving $10.2 \pm$ 3.0 METS and reaching $97 \pm 11 \%$ of the age-predicted maximum heart rate. After exercise termination, stress cine imaging was completed within $47.7 \pm 16.7 \mathrm{sec}$ and stress perfusion by $91.0 \pm 33.6 \mathrm{sec}$. Compared to angiography, exercise CMR demonstrated sensitivity of $86 \%$, specificity of $100 \%$, positive predictive value of $100 \%$ and negative predictive value of $98 \%$. There was strong agreement between treadmill stress CMR and angiography $(\kappa=0.91)$, but only moderate agreement between stress SPECT and angiography $(\kappa=0.55)$ and between CMR and SPECT $(\kappa=0.48)$. At 12 -month follow-up, no major adverse cardiac events had occurred in this cohort of ambulatory patients referred for stress imaging.

\section{Conclusions}

The multicenter EXACT trial indicates both diagnostic and prognostic value of treadmill stress CMR in typical patients referred for exercise SPECT.

${ }^{1}$ Ohio State University, Columbus, OH, USA

Full list of author information is available at the end of the article 


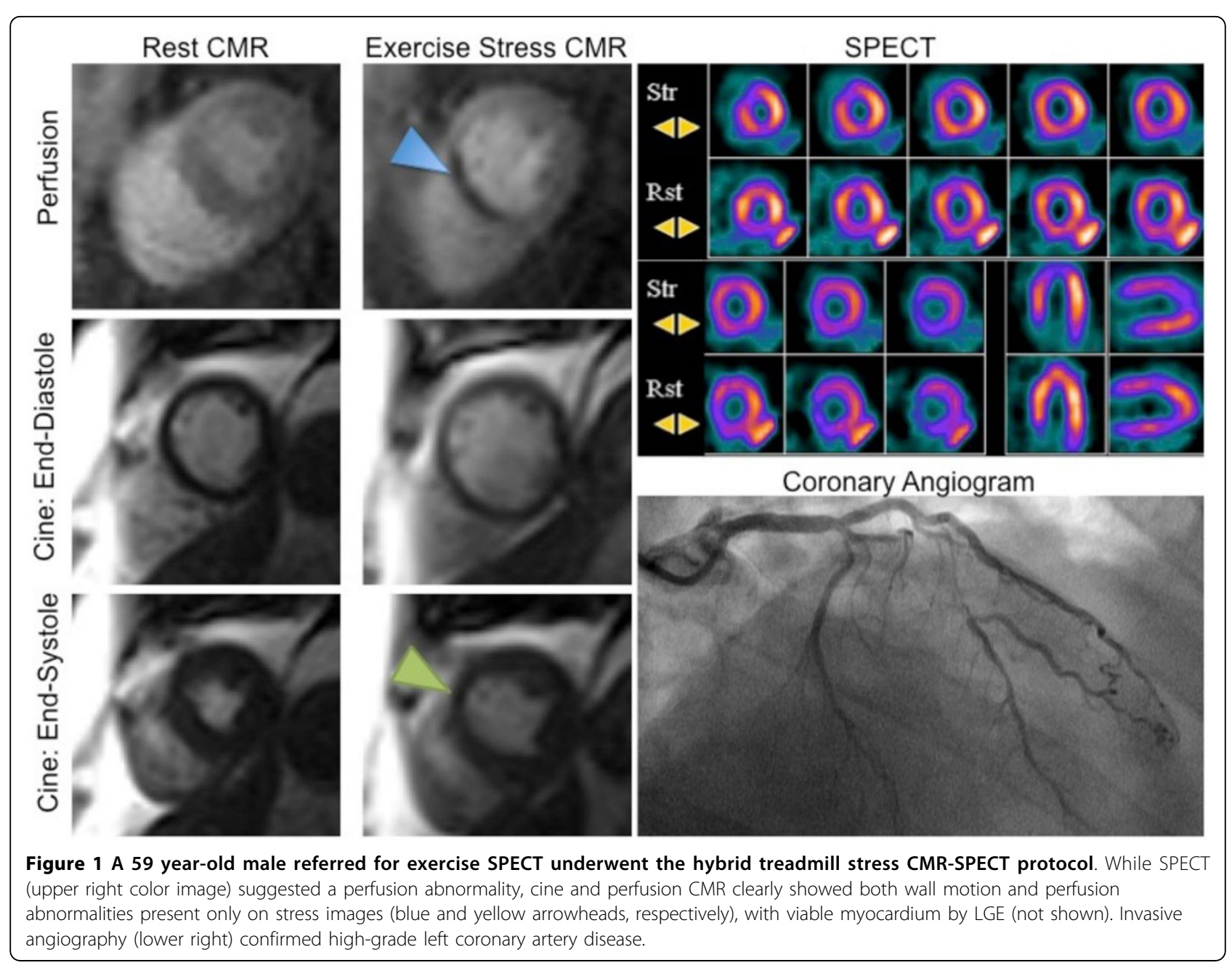

\section{Authors' details}

'Ohio State University, Columbus, OH, USA. ${ }^{2}$ The Christ Hospital, Cincinnati, $\mathrm{OH}$, USA. ${ }^{3}$ University of Pittsburgh, Pittsburgh, PA, USA. ${ }^{4}$ Weill-Cornell Medical College, New York, NY, USA. ${ }^{5}$ Case Western Reserve, Cleveland, $\mathrm{OH}$ USA.

Published: 27 January 2016

doi:10.1186/1532-429X-18-S1-0132

Cite this article as: Raman et al:: Diagnostic performance of treadmill exercise cardiac magnetic resonance: the prospective, multicenter EXACT trial. Journal of Cardiovascular Magnetic Resonance 2016 18(Suppl 1):0132.

\section{Submit your next manuscript to BioMed Central} and take full advantage of:

- Convenient online submission

- Thorough peer review

- No space constraints or color figure charges

- Immediate publication on acceptance

- Inclusion in PubMed, CAS, Scopus and Google Scholar

- Research which is freely available for redistribution 